\title{
Können wir das?
}

\section{Liebe Leserinnen und Leser,}

die Anforderungen an Controller haben sich über die Jahre schleichend, aber im Ergebnis dramatisch verändert. Heute werden regelmäßig nicht nur eine solide Instrumentenkenntnis und analytische Fähigkeiten, sondern auch kommunikative und soziale Fähigkeiten, ein ausgeprägtes Rückgrat und schließlich ein solides Verständnis des zugrunde liegenden Geschäfts erwartet. Zudem hat sich das, was man unter analytischen Fähigkeiten versteht, durchaus mit verändert. Damit ist eben nicht mehr nur rechnen können gemeint, sondern nicht zuletzt auch die Fähigkeit, unscharf konturierte Probleme strukturiert anzugehen. Leider werden die damit verbundenen Fähigkeiten in der traditionellen Lehre im Regelfall nicht systematisch vermittelt. Universitäre Lehre im Controlling beschränkt sich viel zu häufig auf die Vermittlung von Instrumenten und theoretischen Konzepten. Berufspraktische Fähigkeiten kommen dabei zu kurz. Und auch in der Unternehmenspraxis stehen Fähigkeiten wie die strukturierte Problemlösung nicht auf jedem Trainingsplan. Die Vermittlung entsprechender Kompetenzen bleibt in der Regel einführenden Trainings von Strategieberatern und dem „Learning by Doing“ im Berufsalltag vorbehalten. Aber warum eigentlich?

Mit dem vorliegenden Heft wollen wir Sie für das Themenfeld der strukturierten Problemlösung und Kommunikation sensibilisieren. Dabei gibt es eine Vielzahl spannender Aspekte zu beachten: Wie definiere ich überhaupt präzise ein Problem, wenn ich mich nicht mit einem Schlagwort wie Finanzoder Flüchtlingskrise zufriedengeben will? Wie unterscheide ich die Symptome eines Problems von den dahinterliegenden Ursachen? Wie schäle ich die in einem Projekt zu beantwortende Kernfrage präzise heraus? Wie kläre ich möglichst frühzeitig (und systematisch) die Rahmenbedingungen der Problemlösung - man denke nur an Restriktionen auf der InputSeite, aber auch im erlaubten Lösungsraum? Wie kläre ich frühzeitig, was den Erfolg des Projekts definiert? Wie strukturiere ich ein definiertes Problem am besten mithilfe von Entscheidungsbäumen? Wie überführe ich eine entsprechende Struktur in eine umsetzbare Roadmap? Wie priorisiere ich die Schwerpunkte meiner Projektarbeit? Diese Aufzählung

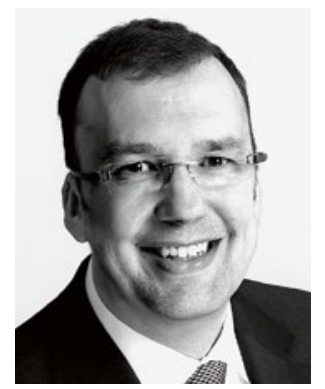

Utz Schäffer

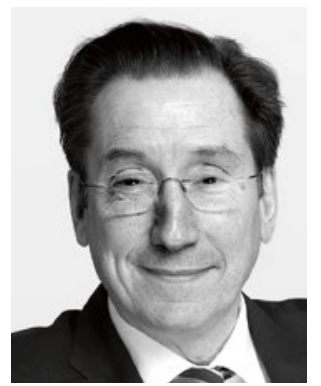

Jürgen Weber ließe sich fortsetzen. Liegt aber ein Lösungsvorschlag für das zugrunde liegende Problem vor, geht die Arbeit vielfach erst richtig los. Es gilt, den Lösungsansatz strukturiert zu kommunizieren und an den Entscheider zu bringen. Just hier liegt auch der Schwerpunkt unseres Hefts. Dabei geht es nicht (nur) um schöne Schaubilder, sondern um die Frage, welche Techniken eine lösungsorientierte und adressatengerechte Kommunikation unterstützen können, wie die interne Konsistenz einer Präsentation sichergestellt werden kann, wie unnötige Barrieren im Kommunikationsprozess vermieden werden und ähnliche Dinge mehr.

Nimmt man als Controller die Herausforderung des Business Partnerings ernst, kann man sich um diese Fragestellungen nicht herumdrücken und sollte vielleicht auch nicht zu vorschnell darauf vertrauen, dass man solche Dinge schon irgendwie „im Griff“ hat. Wir hoffen, dass Ihnen das vorliegende Heft die eine oder andere Anregung mitgibt, und wünschen Ihnen viel Spaß bei der Lektüre.
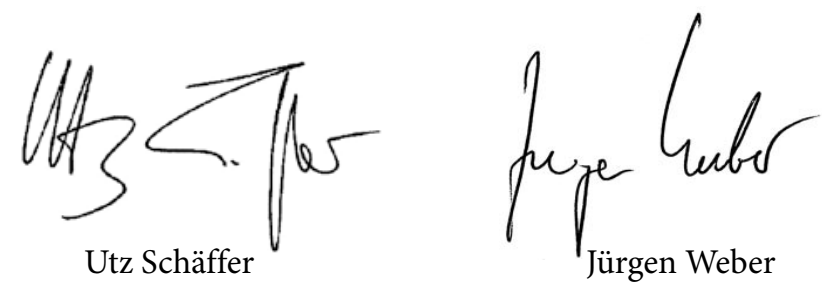